\title{
Electronic Packaging and Reduction in Modelling Time Using Domain Decomposition
}

\author{
Peter Chow ${ }^{1}$ and Choi-Hong Lai ${ }^{2}$ \\ 1 Fujitsu Laboratories of Europe Ltd, Physical and Life Sciences \\ (P.Chow@fle.fujitsu.com) \\ 2 School of Computing and Mathematical Sciences, University of Greenwich \\ (C.H.Lai@gre.ac.uk)
}

Summary. The domain decomposition method is directed to electronic packaging simulation in this article. The objective is to address the entire simulation process chain, to alleviate user interactions where they are heavy to mechanization by component approach to streamline the model simulation process.

\section{Introduction}

Small is exquisite and cool to the consumers of electronic products, but it has enormous technical challenges that need to be overcome by designers and engineers. Elements such as health and safety compliance, power and heat management, and usability are commonly top on the list of issues. The primary technical challenges (Chow and Addison [2002]) are: 1) High density of components leads to an increase of model complexity that needs to address, including any interactions and interferences between the processes. 2) Creating highly intricate and detailed geometry and mesh models with parts of dissimilar scales in appropriate time, for examples, the entire electronic components in a laptop computer and exposure analysis of electronic devices on an entire human body and tissues. 3) Size of computational demands million plus cells/elements models are common in industrial simulations and models with tens of millions of elements are becoming more frequent. 4) The market demands add to the ever-increasing pressures on engineers to speed up the modelling and design cycles.

Whilst domain decomposition has been widely applied to areas such as parallel solvers and preconditioners, coupling of different numerical methods and physical models, it has not been considered for the entire simulation process chain in order to achieve a comprehensive reduction in modelling time. An early concept of using domain decomposition methods in the reduction of modelling time can be found in Chow and Addison [2002]. This paper gives a rigorous approach of the concept and uses the framework of a defect equation 
in the coupling (C.-H. Lai and Pericleous [1997]). Algorithms developed in sections below concentrate on problems with geometrical multi-scale at the macroscopic mathematical models. Numerical experiments, including monophase and multi-phase, are examined with efficiency of the algorithm being linked to the overall modelling time.

\section{Component Approach and Concept}

A basic conventional simulation process chain would look something like: Geometry-Meshing-Analysis-Visualization. An alteration to the model at any stage means going back to the Geometry stage and repeating the procedure. For large and complex models re-do it all is time consuming. A smart way is to take a component based approach where only the altered components are being re-done and thus significantly more efficient. This is generally not possible due to conformal mesh constraint, one continuous volume mesh, in mesh generation and analysis stages. In instances where it is possible, it is frequently for special purposes and non-standard in nature. While in user interaction intensity, geometry creation and meshing are the most user intensive stages, whereas analysis stage is the least, and visualization is interpreting the solution for specific requirements. The concept detailed below is component based with each component independently created, meshed and solved. The model solution is reached when interface conditions between components agrees, this is attained by iterations with the domain decomposition method.

The component meshing and gluing (CMG) approach(Chow and Addison [2002]), takes an approach the same as manufacturing products are assembled A product is a collection of assembled components or parts, connected and bonded together, and commonly, the parts themselves are products produced and marketed by others. This component nesting is the base of CMG and let existing models to be reused for other models. This kind of model assembly is probably most suited to applications where models are constructed from a few basic shapes such as multi-chip module models in electronic packaging. Here, it can be realized by a database of components with simple tools that uses parametric to define basic objects relative to parameters such as length, thickness, mesh density, etc., for rapid primitive component creation.

The model of assembled components is then glue together by either merging components to create a single knitted mesh model or collaborating components using an iterative domain decomposition method. The former methodology requires unstructured meshes or the use of polyhedral type elements to combine into one mesh model, and we will refer to this as the CMG-Knitted strategy in this paper. One disadvantage of the knitted strategy is that it does not apply to all solvers, for example, structured mesh solvers. Only solvers with polyhedral element capability can be considered. The latter methodology requires the domain decomposition method (DDM) method to attain the model solution through the exchange of boundary conditions between 
common interfaces that the components shared. We will refer to this as the CMG-DDM strategy in this paper. The solution of each component may be obtained by means of existing fast solvers. This is more universally applicable to all types of solvers, but one known disadvantage is that the computing time to achieved a converged solution is longer. Fast iterative methods in domain decomposition can significantly shorten the time to solution but it is unlikely to match the knitted mesh case. For appropriate solvers, a combination of the two gluing strategies is possible.

The significant benefit of CMG is it virtually removes all the difficulties commonly associated with model creation and mesh generation which made the two processes extremely manpower intensive in the process chain. And with the volume-mesh generation element no longer called, a considerable saving in time and computing resources. Perhaps the only meshing related element that may need some manpower input are the interface regions where the component meets. This is not envisage, but if needed, it is a surface meshing problem and not a volume one which is one-degree of dimension less in complexity and requires significantly less computing to do. This gain needs to be summed with the increase computing times in the analysis stage expected in the CMG-DDM strategy to give an account of profit or lost balance. When the balance is at a significant lost we do have the parallel processing option to address the problem. Compared to model creation and mesh generation, parallel processing has drastically cut the time for the analysis element and the trend is still downward.

\section{Numerical Algorithms}

Provided that the solvers can take polyhedral elements, the CMG-Knitted strategy does not require extra effort to put into the solvers. It is the meshmodel that needs to be knitted at the finite-element mesh topology level, gluing the interfaces of the mesh components. In the CMG-DDM strategy, the domain decomposition method (DDM) (Chow and Addison [2002]) is ideally suited for the assembled-component model, with the non-overlapping class the most appropriate. A non-overlapping approach allows flexibility in the mesh processing, the methods of numerical solution, the handling of different physics, and the adoption of numerical solvers in each of the model components. This choice also makes the defect equation technique as developed in C.-H. Lai and Pericleous [1997] an ideal method for CMG-DDM.

Let $\mathcal{L} u=f$ be defined in the domain $\Omega$ and $u=g$ on $\partial \Omega$, where $\mathcal{L}$ may be a nonlinear operator that depends on $u$, and $g$ is a known function. The domain $\Omega$ is partitioned into $M$ non-overlapped sub-domains such that $\bigcup_{i=1}^{M} \Omega_{i}=\Omega$ and $\Omega_{i} \cap \Omega_{j}=$, for $i \neq j$. Each sub-domain is associated with a sub-model defined by $\mathcal{L}_{i} u_{i}=f_{i}$. The boundary of each sub-domain, $\partial \Omega_{i}$, subtracting the part of boundary which overlaps with the boundary of the entire problem is in essence a part of the interface. Therefore the interface, which attached to 
$\Omega_{i}$, may be defined as $\gamma_{i}=\partial \Omega_{i} \partial \Omega$. The boundary conditions defined on $\gamma_{i}$ may be denoted by $u_{\gamma}$ and it satisfies a defect equation, such as $D\left(u_{\gamma}\right)=0$ (C.-H. Lai and Pericleous [1997]). Using superscripts to denote the number of gluing process, the CMG-DDM algorithm may be written as follows.

Initial values: $n=0 ; u_{i}^{(0)}, i=1, \ldots, M$ are given.

Repeat $\{n:=n+1$;

Do $i=1, \ldots, M$

$u_{i}^{(n)}:=\left\{\right.$ Solve $\mathcal{L}_{i} u_{i}^{(n)}=f_{i}$ in $\left.\Omega_{i}\right\} ;$

subject to:

End-Do

$u_{i}^{(n)}=g$ on $\partial \Omega \bigcap \partial \Omega_{i}$ and $\left.u_{i}^{(n)}\right|_{\gamma_{i}}=u_{\gamma_{i}} ;$

Until $\|D\|_{2}<\epsilon$

When the model consists of a single domain (meshed component) then the For loop and the defect calculation, $D\left(u_{\gamma}\right)=0$, are redundant. The CMG-Coupled cases are performed in this way. From the above algorithm the For loop may be run in parallel and on homogeneous computing systems the solution are identical between parallel and scalar computations.

\section{Numerical Experiments}

The particular problem to be considered in this paper is governed by the 2-D energy equation, limited to conduction only, in temperature $u$. The variables in $(2)$ are density $(\rho)$, specific heat $(c)$, thermal conductivity $(k)$, time $(t)$ and the source term $(S)$.

$$
\rho c \frac{\partial u}{\partial t}=\nabla \cdot(k \nabla u)+S(u)
$$

The nonlinearity is introduced in the form of a material phase-change in the source term. For solidification using the enthalpy source-based method this is given by

$$
S(u)=L \rho \frac{\partial f(u)}{\partial t}
$$

where $L$ is the latent heat and $f$ is the liquid fraction. The algorithm for solving these kinds of problems may be found in papers by Chow and Cross [1992] and Voller and Swaminathan [1991] and is not discussed in this paper. Readers interested in obtaining more information are directed to these references. In this study, the numerical stable method of Voller and Prakash [1987] solidification algorithm is used.

A nonlinear problem with phase-change occurring inside the domain, geometry as that of Fig. 1, was used to conduct the numerical experiments and investigations. Three experiments conducted were: 1) A steady state heat transfer (no phase-change) where the top surface is at a temperature of $10^{\circ} \mathrm{C}$ 
and bottom surface of $100^{\circ} \mathrm{C}$. The left side of the model is symmetry and for all other boundaries, a convective heat boundary condition of ambient temperature of $25^{\circ} \mathrm{C}$ with a heat transfer coefficient of $10.0 \mathrm{~W} / \mathrm{m}^{2} \mathrm{C}$. 2) A transient heat transfer problem that has the same boundary conditions as the first experiment with an initial temperature of $100^{\circ} \mathrm{C}$. The time step size taken was 10 seconds interval and simulation time end at 120 seconds. 3) The final experiment is a heat transfer with the small solder bumps (Ch4 Solder Bump in Fig. 1 undergoing solidification. The boundary condition is essentially the same as the previous two experiments with both top and bottom surfaces now have the convective heat boundary conditions. The initial temperature is at $183^{\circ} \mathrm{C}$ with time step size of 2 seconds interval and simulation time end at 600 seconds.

Table 1 shows the dimension of the components and Table 2 shows the material properties data used in the experiments. For convenient, the Si-Chip, MCM-L and motherboard take on the material property of the Board dataset, and both the Ch4 and BGA solder bumps take on the Solder dataset. For the third experiment, only the Ch4 solder bumps are solidifying, the liquidus and solidus temperatures for BGA solder bumps are set above that given thus no solidification occurs.

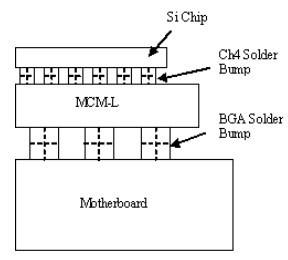

Fig. 1. An example of multiple chip model geometry.

Table 1. Geometric dimension of components in the test model.

\begin{tabular}{l|ccc}
\hline & Length $(\mathrm{mm})$ & Height $(\mathrm{mm})$ & Gap Interval $(\mathrm{mm})$ \\
\hline Si Chip & 10.5 & 1.5 & \\
Ch4 Solder Bump & 1.0 & 1.0 & 1.0 \\
MCM-4 & 15.5 & 2.0 & 3.0 \\
BGA Solder Bump & 2.0 & 2.0 & \\
Motherboard & 19.5 & 3.0 & \\
\hline
\end{tabular}

Fig. 2 shows two different meshes used in present experiments. Figures 3 to 5 show the cell invariant temperature distribution of the CMG-Knitted computation and CMG-DDM for the three experiments. The temperature profile on the two meshes (conformal and non-conformal) is virtually the same. Ta- 
Table 2. Material properties.

\begin{tabular}{|c|c|c|c|c|c|c|}
\hline & $\mid \begin{array}{l}\text { Density } \\
\mathrm{kg} / \mathrm{m}^{3}\end{array}$ & $\begin{array}{c}\text { Specific } \\
\text { Heat } \\
\mathrm{J} / \mathrm{kg} \mathrm{C}\end{array}$ & $\begin{array}{l}\text { onductivit } \\
\mathrm{W} / \mathrm{m} \mathrm{C}\end{array}$ & $\begin{array}{l}\text { Liquidu } \\
\text { Temp. } \\
\text { C }\end{array}$ & $\begin{array}{c}\text { Solidus } \\
\text { Temp. } \\
\text { C }\end{array}$ & $\begin{array}{c}\text { Latent } \\
\text { Heat } \\
\mathrm{J} / \mathrm{kg}\end{array}$ \\
\hline Board & 1400 & 838 & 0.18 & & & \\
\hline Solder & 8400 & 171 & 50.6 & 183 & 180 & 3700 \\
\hline
\end{tabular}

ble 3 give the total energy in the system domain and computing costs for the simulation, together with iteration numbers required. The knitted conformal mesh result is used as the reference guide towards measuring accuracy and computing performance. In the transient problems, the iteration numbers shows the first time step has the highest iteration counts, this is obvious due to the cold starting the simulation, whereas lowest is found in time steps towards end of simulation. The CMG-knitted computation for linear problems, Experiments 1 and 2, require 2 iterations for both steady state and per time step in transient to achieve convergence on temperature. The largest deviation of the solution from the referenced data is under $0.2 \%$ in Experiment 3, and under $0.07 \%$ and $0.01 \%$ respectively for Experiments 2 and 1. Computing times for CMG-DDM for the two meshes (conformal and non-conformal) are 72.1 and 65.0 in Experiment 1, 44.5 and 40.2 in Experiment 2, and 2.1 and 2.0 in Experiment 3, times more expensive respective to the referenced knitted conformal mesh cases.

Based on these results the CMG-DDM approach for linear problem is not competitive, but non-linear problem is a different proposition. Assuming 25\% of overall time is used for analysis, this implies the projected total modelling time of 197 seconds $(=49.296 / 25 \%)$ for coupled computation in Experiment 3 , which suggests that the CMG-DDM approach to be competitive in nonlinear phase-change problems in electronic packaging.

\section{Summary}

Numerical experiments conducted indicates potential advantages of the CMG method in electronic packaging for non-linear solder solidification based on the enthalpy method (Chow and Cross [1992], Voller and Prakash [1987]) simulation of multiple chip modules. The success of the method is the mechanization by component approach to streamline the model simulation process at model creation and mesh generation stages which are the most manpower intensive.

\section{References}

A. C. C.-H. Lai and K. Pericleous. A defect equation approach for the coupling of subdomains in domain decomposition methods. Computers Math. 
Applic., 6:81-94, 1997.

P. Chow and C. Addison. Putting domain decomposition at the heart of a mesh-based simulation process. Int. J. Numer. Meth. Fluids, 40:1471-1484, 2002.

P. Chow and M. Cross. An enthalpy control volume-unstructured mesh (cvum) algorithm for solidification by conduction only. Int. J. Numer. Meth. in Engg., 35:1849-1870, 1992.

V. Voller and C. Prakash. A fixed grid numerical modelling methodology for convection-diffusion mushy region phase change problems. Int. J. of Heat and Mass Transfer, 30:1709-1719, 1987.

V. Voller and C. Swaminathan. General source-based methods for solidificationn phase change. Numerical Heat Transfer, 19:175-190, 1991.

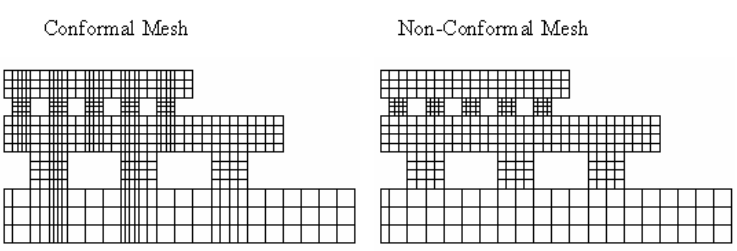

Fig. 2. Two different meshes used in experiments.

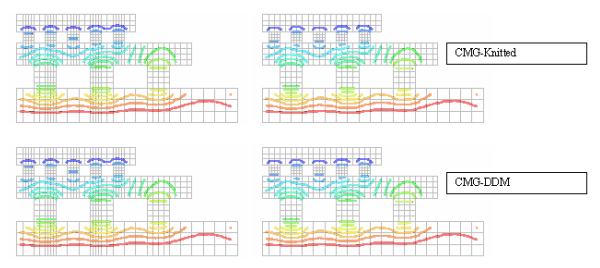

Fig. 3. Comparison of the temperature distribution of steady state results.

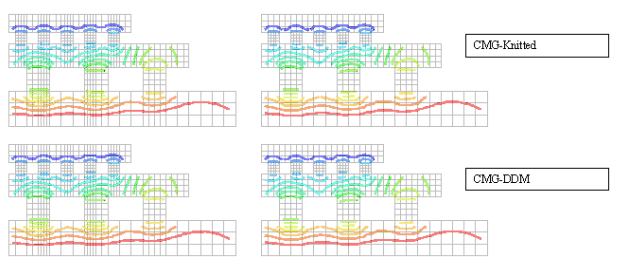

Fig. 4. Comparison of the temperature distribution of transient results. 


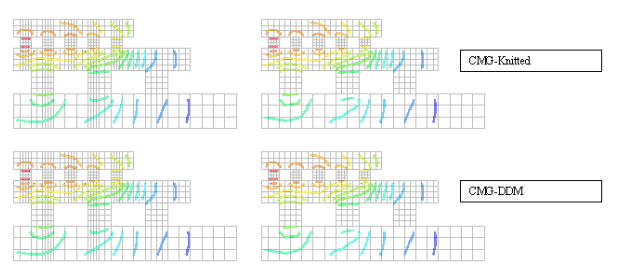

Fig. 5. Comparison of the temperature distribution of solidification results.

Table 3. Total energy in system and computing times (Computing platform: P4, $2 \mathrm{GHz}, 1 \mathrm{~GB} \mathrm{RAM})$.

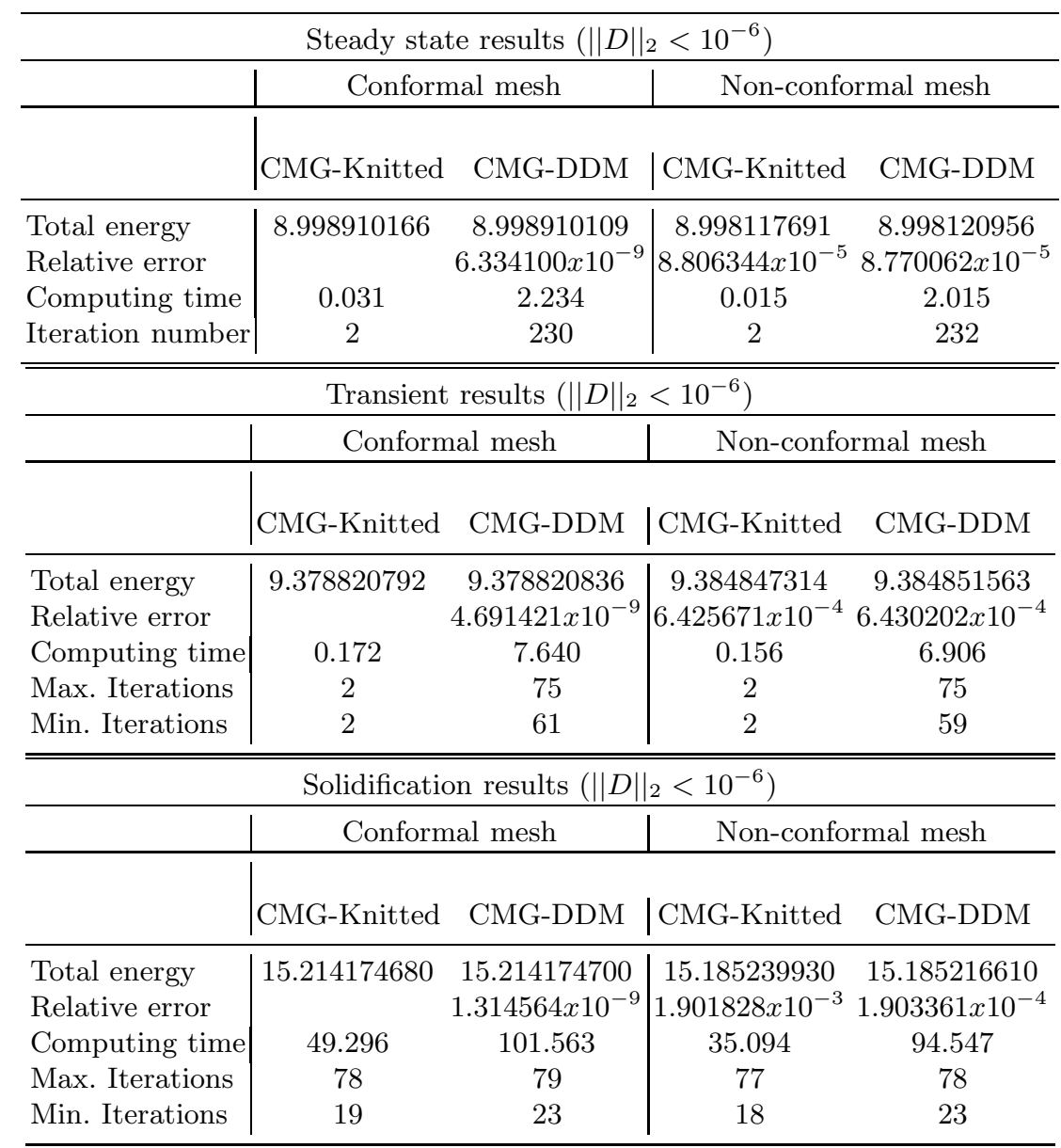

